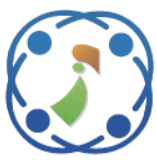

\title{
Genre Classification of Telugu and English Movie Based on the Hierarchical Attention Neural Network
}

\author{
Kumar Reddy Govindaswamy ${ }^{1 *}$ \\ Shriram Ragunathan ${ }^{1}$ \\ ${ }^{1}$ Department of Computer Science \& Engineering, Bharathiar University, India \\ * Corresponding author's Email: rgkumarresearchscholar@gmail.com
}

\begin{abstract}
Genre Classification of movies is useful in the movie recommendation system for video streaming applications like Amazon, Netflix, etc. The existing methods used either video or audio data as input that requires more computation resources to process the data for the genre classification of movies. In this study, the Hierarchical Attention Neural Network (HANN) is proposed for genre classification of movies based on the social media called Twitter data as input. Twitter data related to the Telugu and English movies are collected and applied to HANN for movie's genre classification. IMDB data are used to evaluate the performance of the proposed HANN method. The hierarchical structures of the twitter data is considered by the proposed HANN method and the most important words related to genre classification is identified by the attention mechanism, where the other neural networks such as Artificial Neural Network and Convolutional Neural Network (CNN) returns only the important weights resulting from previous words. The HANN method has the advantages of encoding the relevant information that helps to improve the performance of the recommendation system. The experimental results show that the HANN method achieve higher performance compared to other classifiers Long Short-Term Memory (LSTM) and Bidirectional LSTM (Bi-LSTM). The HANN method achieves accuracy of $73.15 \%$ in classification, while the existing BiLSTM method achieve the accuracy of $68 \%$ in classification.
\end{abstract}

Keywords: Hierarchical attention neural network, Long short term memory, Movie genre classification, Recommendation system, Telugu movies.

\section{Introduction}

Accurate movie genre classification is essential in the movie recommendation system for video streaming services like Netflix, Amazon prime videos, etc. to reduce computational cost [1]. As the competition increases in the video streaming market, they require a robust recommendation system to increase the profit and customer's satisfaction. The recommendation system suggests movies to the users based on their previous watch history, genre interest and purchase records [2]. The existing methods on genre classification are based on the video or audio input data that requires more computation resources to process the data [3, 4]. Sentiment analysis is the process of analyzing the user's preferences based on the social media that can be applied for genre classification of text data. The user can freely express their views, opinions and feelings on the events or movies through their tweets [5]. Sentiment analysis involves in the lexicon based method and machine learning methods that can be used for the classification [6].

In recent years, the researchers implemented the machine learning and deep learning techniques for the sentiment analysis, but only the deep learning methods showed the efficient performance. Deep learning methods such as CNN, LSTM and BiLSTM provide the effective performance due to learning capabilities of these techniques [7]. In India, digital content in the Indian language is growing rapidly due to easy accessibility of internet and language modelling. Therefore, a lot of researches are required in the computational linguistics for solving real world problems in the native linguistic use $[8,9]$. The existing genre classification method is based on the video and audio data that requires more 
computational resources [10]. In this study, the HANN method is proposed for the movie genre classification based on twitter data. In order to improve the performance of the HANN method, the relevant information is encoded and this relevant information are returned by the encoder and the importance weights of these data as one vector is computed by attention mechanism. The overfitting issues are also considered by the proposed HANN method and it is compared with other classifiers namely Support Vector Machine (SVM), Random Forest (RF), LSTM and BiLSTM. The results show that the HANN method has achieved higher performance compared to the other classifiers.

The paper is organized as the literature review is presented in the Section 2, the description about the proposed HANN method is presented in Section 3, the experimental setup is discussed in Section 4, the results are provided in Section 5 and the conclusion is drawn in Section 6.

\section{Literature review}

Sentiment analysis is an interesting topic in the Natural Language Processing (NLP) that applies to the opinion mining. Recent researches related to the Sentiment analysis and movie genre classification have been reviewed in this section.

Wei, Liao, Yang, Wang and Zhao [11] proposed BiLSTM model with Multi-polarity investigation for sentiment analysis. The difference between the words and the sentiment orientation were analyzed by the method for Multi-polarity analysis. This difference was considered as the important information for the sentiment analysis. During optimization, the performance of discriminatory was improved by an orthogonal restriction mechanism. The experimental result on dataset showed that the BiLSTM method achieved higher effectiveness in analyzing the sentiment polarities. However, the external knowledge base is not included in this model, which leads to the poor performance of the method.

$\mathrm{Li}, \mathrm{Cui}$, Shen and Ma [12] proposed a method that mined the user's preference based on the social media to evaluate the similarity between the online movies and TV episodes. Based on this method, cold start problem was effectively solved. A series of data mining method and social computation models were adopted in this method. Twitter data were used to analyze the performance of the method. The developed method achieved higher efficiency in the movie recommendation system. However, the LSTM method can be adopted to increase the performance of the developed model.
Nguyen and Nguyen [13] proposed a combination of LSTM and CNN in the sentiment analysis. CNN was employed in the filters to capture local dependencies and the LSTM was used to store the information for the long term. The freezing technique was used in this method to avoid the overfitting problem in deep learning. The experimental results showed that the developed model achieved higher performance in the sentiment analysis. The Naïve Bayes with SVM (NBSVM) model was also added in a neural voting ensemble to boost the performance. However, the similarity values were not calculated to form the clusters of NBSVM.

Wehrmann and Barros [14] performed the genre classification for multi-label movie trailer by developing the CNN based architecture. The architecture was developed for an Ultra-deep CNN with residual connections and temporal information are extracted by this technique, which was a kind of special convolution layer. The experimental analysis showed that the proposed CNN method outperformed existing method in movie genre classification. This study uses the video data for the genre classification, but text data can be used to increase the computation process.

Chen, $\mathrm{Xu}, \mathrm{He}$ and Wang [15] proposed a divide and conquer method to classify the sentence into various types and sentiment analysis was performed on each type. According to the number of targets, the sentences were classified using the Neural Network based sequence model. One dimensional CNN obtained each class of sentence as input for final classification. The BiLSTM with Conditional Random Fields (BiLSTM-CRF) method is applied to the sentence for classification based on the number of targets. The performance of the sentence-level sentiment was improved by sentence type classification. However, the sequence learning model can be applied to improve the performance of this method on another language.

Wi, Jang and Kim [16] developed a gram layer in CNN to extract the optimal information from the movie posters for final classification. A feature map was created by applying the gram matrix with style features. The method created the poster dataset into 12-multi-genres for movie genre classification. The results pointed out that gram layer in $\mathrm{CNN}$ achieved better performance compared to residual neural network. However, the method is insufficient to handle the imbalance dataset of rare genres, as the number of defined genres was limited to 12 .

Shambharkar and Doja [17] implemented the optimized Deep CNN (DCNN) for human action based movie classification using video sequences. The pre-processing was conducted using adaptive 
median filtering process and the foreground portions were extracted using the threshold based segmentation approach. The visual features and motion features were combined in the feature extraction stage and provided as input to DCNN for classification. The experiments were conducted to test the efficiency of the developed method in terms of false alarm rate, precision, accuracy, recall, fmeasure and false discovery rate. However, this method misclassifies samples due to ineffectiveness of filtering techniques.

The existing methods in genre classification are based on the video and audio data that requires more computation resources. In this research study, text data are applied in the genre classification to increase the performance of the proposed HANN method.

\section{Proposed method}

Movie Genre Classification is required in the video streaming services for movie recommendation systems. Though, existing methods for movie genre classification are based on the video and audio. So, existing method requires more computation resources that reduce performance of the system. This study implements a movie genre classification method based on the text data. Data of last year Telugu movies from the IMDB with their genre and twitter data related to each movie are collected. Hierarchical Attention Neural Network (HANN) is used for the movie genre classification. The data collected from the IMDB genre were used for the ground truth to test the performance of the developed method. The Fig. 1 presents the block diagram of the proposed HANN method.

\subsection{Hierarchical attention networks}

A hierarchical attention network [18] is adopted in the document representation in this study. The architecture is generally in hierarchical structure, but contains different kinds of components, i.e. encoder and attention models. Twitter data are denoted as $D=\left\{\left(x_{i}, y_{i}\right), i=1, \ldots, N\right\}$ consist of $N$ number of tweets related to movies $x_{i}$ with labels $y_{i} \in$ $\{0,1\}^{k}$. Each document is grouped into sentences, $x_{i}=\left\{w_{11}, w_{12}, \ldots, w_{K T}\right\}$, where every document is described as the sequence of $d$-dimensional embedding of their words, the maximum number of words is represented as $T$ in a sentence and the maximum number of sentences is depicted as $K$ in a document.

The model considers the input as twitter data $x_{i}$ and output the class as $u_{i}$. This consists of two levels of abstraction, sentence vs. word. The similarity of word level is analyzed by the method and it consists of an attention model $a_{w}$ with parameter $A_{w}$ and an encoder $g_{w}$ with parameter $H_{w}$. The classification layer uses the output as $u_{i}$ to measure $y_{i}$.

\subsubsection{Encoder layer}

In the word level, the sequence of input words $\left\{w_{i t} \mid t=1, \ldots, K T\right\}$ are encoded in the function $g_{w}$ for each sentence $i$ is presented in Eq. (1).

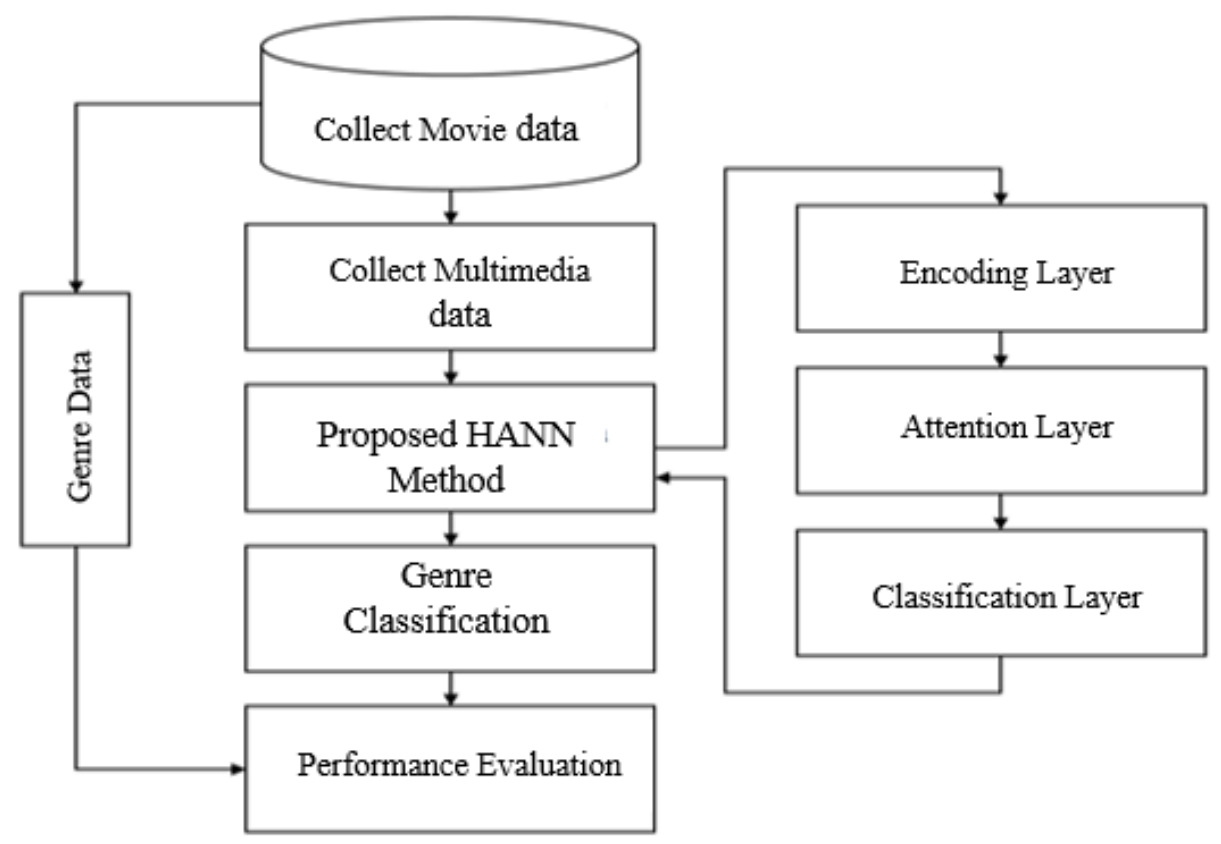

Figure. 1 The block diagram of proposed HANN in genre classification 


$$
h_{w}^{i t}=\left\{g_{w}\left(w_{i t}\right) \mid t=1, \ldots, K\right\}
$$

At the sentence level, $\left\{h_{w}^{i t} \mid t=1, \ldots, T\right\}$ as intermediate word vectors are combined to $s_{i}$ as a vector sentence. The sequence of sentence vectors $\left\{s_{i} \mid i=1, \ldots, K\right\}$ is developed by the function $g_{s}$, where sentence vectors' sequence is assumed as $h_{s}^{i}$.

In this study, a fully-connected Gated Recurrent Unit (GRU) network [16] known as GRU2 and a bidirectional GRU is used. The temporal information of forward or backward at a time is presented in the bidirectional GRU as BiGRU. After that, a hidden state of every input vector is concatenated to develop this BiGRU, where input vector is obtained from forward GRU $\overrightarrow{g_{w}}$ and backward GRU $\stackrel{\leftarrow}{g_{w}}$, presented in Eq. (2).

$$
h_{w}^{i t}=\left[\overrightarrow{g_{w}}\left(h_{w}^{i t} ; \overleftrightarrow{g_{w}}\left(h_{w}^{i t}\right)\right)\right]
$$

The hidden state presentation uses the same concatenation of a sentence $h_{s}^{i}$.

\subsubsection{Attention layer}

The sequence of input word is described at every level and considered the last hidden-state vector, which is provided by encoder. All relevant information is difficult to encode in a fixed-length vector. Attention layer is introduced at each level to solve this problem and obtain the importance of each hidden state vector to denote the document meaning respectively. The parameter $s_{i} \in R^{d_{w}}$ defines the sentence vector and $d_{w}$ illustrates the word encoder dimension that is calculated using Eq. (3).

$$
\frac{1}{T} \sum_{t=1}^{T} a_{w}^{i t} h_{w}^{i t}=\frac{1}{T} \sum_{t=1}^{T} \frac{\exp \left(v_{i t}^{\mathrm{T}} u_{w}\right)}{\sum_{j} \exp \left(v_{i j}^{\mathrm{T}} u_{w}\right)} h_{w}^{i t}
$$

Where, fully-connected neural network is illustrated as $v_{i t}=f_{w}\left(h_{w}^{i t}\right)$ with $W_{w}$ parameters. Similarly, the document vector $u \in R^{d_{s}}$, where the sentence dimension encoder is described as $d_{s}$ and it is measured using the Eq. (4).

$$
\frac{1}{K} \sum_{i=1}^{K} \alpha_{S}^{i} h_{S}^{i}=\frac{1}{K} \sum_{i=1}^{K} \frac{\exp \left(v_{i}^{\mathrm{T}} u_{S}\right)}{\sum_{j} \exp \left(v_{j}^{T} u_{s}\right)} h_{S}(i)
$$

Where, fully-connected neural network is denoted as $v_{i}=f_{s}\left(h_{s}^{i}\right)$ with $W_{s}$ parameters. The context of word and sentence are encoded with the vectors $u_{w}$ and $u_{s}$ and these parameters are also learned with other parameters. For $a_{w}$, the total set of parameters is $A_{w}=\left\{W_{w}, u_{w}\right\}$ and for $a_{s}$, it is $A_{s}=$ $\left\{W_{s}, u_{s}\right\}$

International Journal of Intelligent Engineering and Systems, Vol.14, No.1, 2021

DOI: $10.22266 /$ ijies2021.0228.06

\subsubsection{Classification layers}

The softmax layer obtains the output of attention layer with loss as input for final classification, where the loss depends on the cross-entropy between the corrected labels' negative log likelihood. But, the probability of the most likely label is overemphasized by softmax layer, which is not applicable for multilabel classification, so more than one genre representation is presented in each tweets. In addition, sigmoid function replaces the softmax layer, hence the vector $u_{i}$ is used to represent the each document $i$ and the probability of the $k$ labels is modelled in the following Eq. (5).

$$
\widehat{y_{l}}=p\left(y \mid u_{i}\right)=\frac{1}{1+e^{-\left(W_{c} u_{i}+b_{c}\right)_{e} \in[0,1]^{k}}}
$$

Where $d_{s} \times k$ matrix represents the $W_{c}$ and $b_{c}$ depicts the classification layers' bias term. According to cross-entropy measurement, the training loss is evaluated using Eq. (6).

$$
\mathcal{L}(\theta)=-\frac{1}{N} \sum_{i=1}^{N} \mathcal{H}\left(y_{i}, \widehat{y_{l}}\right)
$$

Where $\theta$ is notation for model parameters (i.e. $\left.H_{w}, A_{w}, H_{s}, A_{s}, W_{c}\right)$ and for a document $i, H$ denotes the binary cross-entropy of $y_{i}$ and $\widehat{y}_{l}$. In order to improve the performance of classification, training loss is minimized with Adam optimization or stochastic gradient descent (SGD).

\section{Experimental design}

Movie Genre Classification is evaluated using the text data to analyze the efficiency of the genre detection. This section provides a brief description about the datasets, evaluation metrics and comparison method and system specification. The proposed HANN method is tested and compared with existing methods LSTM, BiLSTM, SVM and RF.

Dataset: The Telugu movies and English movies for the past 3 years (2017-2019) are collected from the IMDB along with the genre. Data on the tweet related to the movies are collected from the twitter. The genres of dataset include mystery, horror, $\mathrm{Sci}-\mathrm{Fi}$, adventure, comedy, action, animation, crime, thriller, drama and fantasy. Twitter data is used by the proposed HANN method for detection of movie's genre and evaluated the performance of the proposed method. In order to improve the performance of HANN method, multimedia data such as video and audio are used. Three years (2017 to 2019) data of English and Telugu movies are collected from IMDB and processed for genre classification. 
Performance Metrics: The three metrics Accuracy, Precision and Recall are used to evaluate the performance. The mathematical Eq. (7) shows the formula of precision which is used to measure the true positive data where a portion of positive data is identified by recall for a given cluster is defined in Eq. (8).

$$
\begin{array}{r}
\text { Precision }=\frac{T P}{T P+F P} \times 100 \\
\text { Recall }=\frac{T P}{T P+F N} \times 100
\end{array}
$$

In order to determine the outliers, the measurement of statistical variability and random errors are used to define the overall accuracy, which is presented in Eq. (9)

$$
\text { Accuracy }=\frac{T P+T N}{T P+T N+F P+F N} \times 100
$$

Where, TP is defined as True Positive, TN is denoted as True Negative, FP is illustrated as False Positive and FN is depicted as False Negative in the above three equations.

System Requirement: The proposed HANN method is implemented in the system consists of Intel i5 processor with $8 \mathrm{~GB}$ of RAM and $500 \mathrm{~GB}$ hard disk. Python 3 is used to test the HANN method and Twitter API.

\section{Experimental results}

Movie Genre Classification is essential for video streaming services to recommend movies to user. The existing methods uses the video or audio data as input for the movie genre classification, but this research uses the text data from social media for movie genre classification. In this study, SVM, RF, LSTM and BiLSTM are implemented on the all collected data including audio, video and text. Three years of
Telugu and English movies data are used to evaluate the performance of the HANN method and compared its performance with the existing method's performance. The three metrics Accuracy, Precision and Recall are measured to explore the performance.

\subsection{Quantitative analysis of proposed method on text data}

Genre classification is carried out using the proposed HANN and other classifiers based on twitter data, as present in Fig. 2. It shows that the HANN method achieved higher performance (i.e. $73.15 \%$ of accuracy) in the genre classification. Due to the use of twitter data, the efficiency of the movie genre classification is increased. The BiLSTM method achieves the second highest performance (i.e. $65 \%$ of accuracy) in genre classification. The LSTM method achieves a considerable performance in genre classification. While comparing with the other classifiers namely SVM, LSTM and BiLSTM, RF achieved only $58 \%$ of accuracy on Twitter data. The reason for achieving better performance in HANN method is that the HANN method encodes the relevant information in the genre classification that increases the performance.

Fig. 3 shows the graphical presentation of proposed HANN method with various existing methods such as SVM, LSTM and HANN in terms of precision. The precision value indicates that the HANN method has achieved higher performance (i.e. 93\% of precision) in movie genre classification compared to the other classifiers SVM and RF. The SVM has $82 \%$ of precision and RF has only $81 \%$ of precision on Twitter data. The proposed HANN method gains higher efficiency due to encode of the relevant information. The LSTM and BiLSTM method have also gained higher efficiency in the movie Genre Classification. The proposed HANN

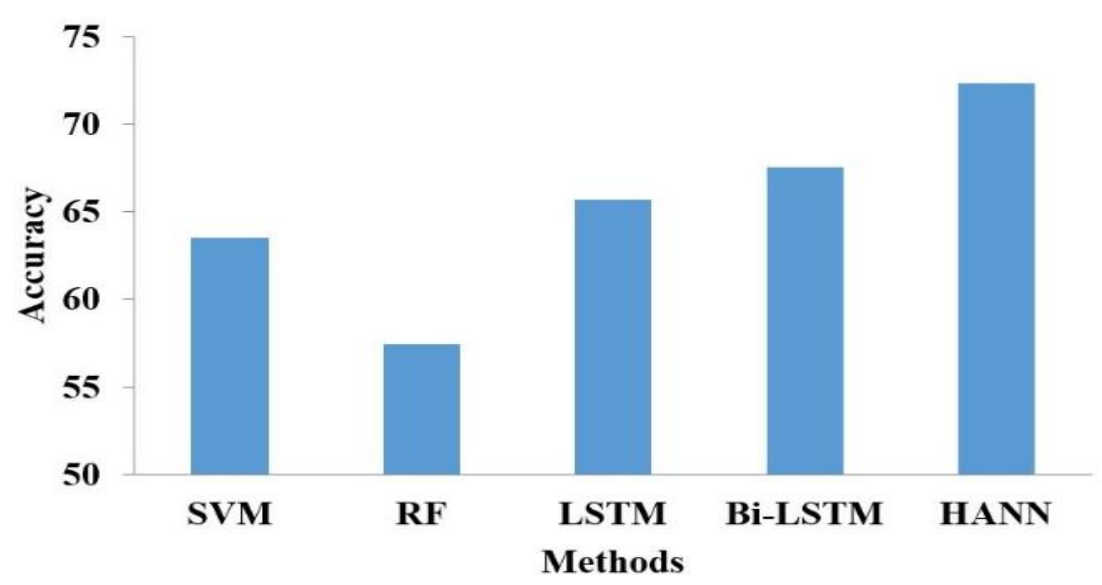

Figure. 2 Accuracy measure of genre classification 


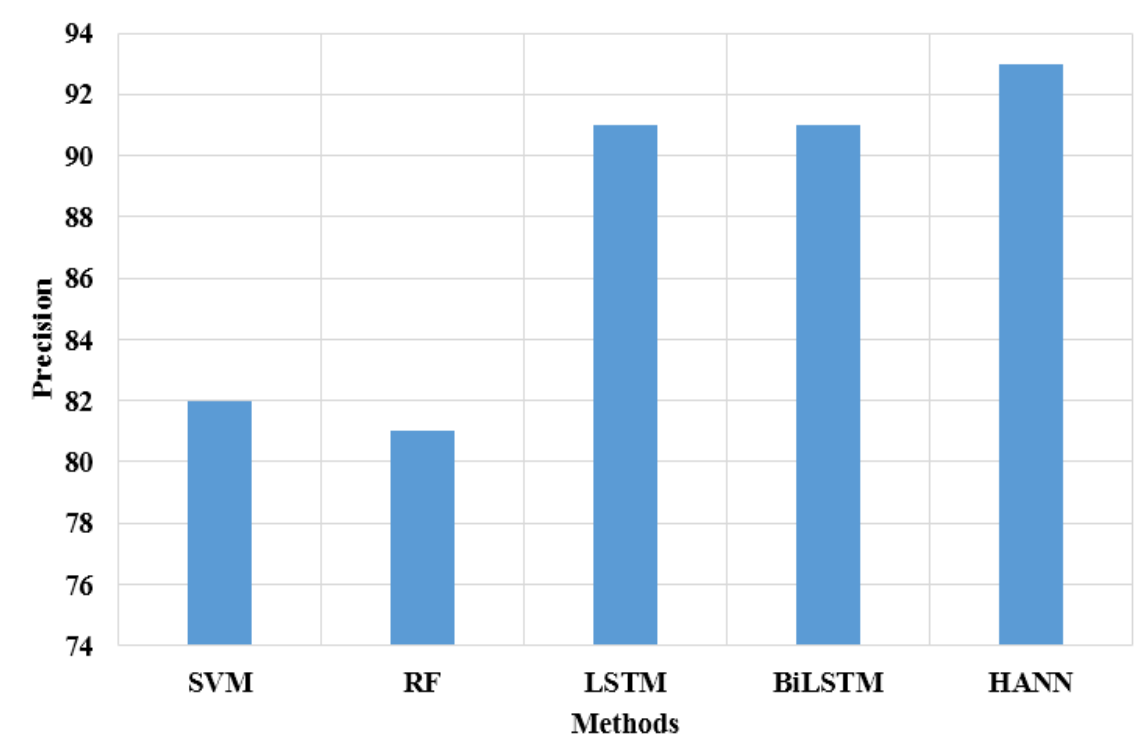

Figure. 3 Precision of genre classification

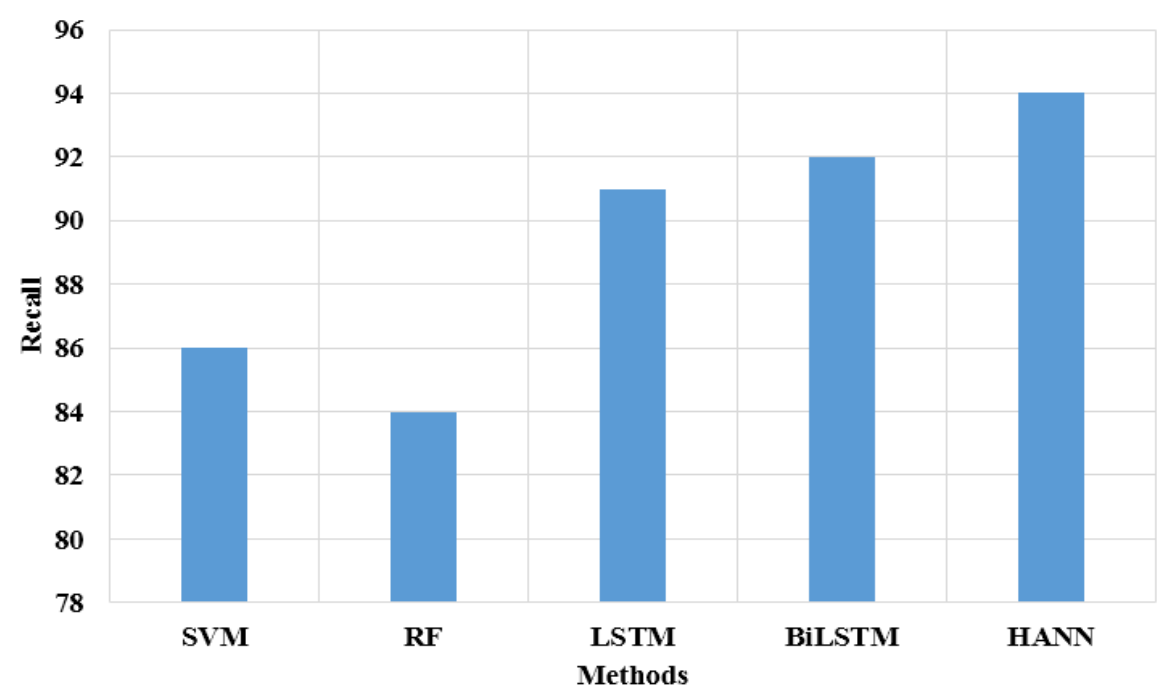

Figure. 4 Recall value of the proposed HANN method in movie genre classification

method achieves $93 \%$ precision and the existing BiLSTM method achieves $91 \%$ precision in classification.

The Fig. 4 presents evaluation of recall for the HANN method and other classifiers are evaluated in movie genre classification. The recall value of the proposed HANN method (i.e. $94 \%$ of recall) is high compared to other classifiers SVM and RF method, because the HANN method able to encode the relevant information in the training process. But, RF is the only classifier, which has low recall (i.e. $84 \%$ of recall) than other all classifiers. The LSTM and BiLSTM method also have the considerable performance in the movie genre classification based on the twitter data. The recall value of the HANN method is $94 \%$, while the existing method of BiLSTM method is $92 \%$.
The results indicate that the proposed HANN method in the movie genre prediction based on the social media data achieves higher efficiency. The proposed HANN method can also use in solving the cold start problem of movie recommendation.

\subsection{Quantitate analysis of proposed HANN method on video data}

In this section, the existing techniques SVM, RF, LSTM and BiLSTM with proposed HANN are implemented on the video data in terms of accuracy, precision and recall for Telugu and English movies genre classification. Table 1 presents the validated results of HANN method with SVM, RF, LSTM and BiLSTM. The value present in the Table 1 indicates that the proposed HANN method achieved better performance on video data in terms of accuracy, 
Table 1. Analysis of different classifiers on video data

\begin{tabular}{|c|c|c|c|}
\hline \multirow{2}{*}{ Methodology } & \multicolumn{3}{|c|}{ Parameters (\%) } \\
\cline { 2 - 4 } & Accuracy & Precision & Recall \\
\hline SVM & 86 & 83.50 & 90 \\
\hline RF & 79 & 82.15 & 85 \\
\hline LSTM & 89 & 91.20 & 91 \\
\hline BiLSTM & 90 & 92.76 & 93 \\
\hline $\begin{array}{c}\text { Proposed } \\
\text { HANN }\end{array}$ & 96 & 95.15 & 96 \\
\hline
\end{tabular}

Table 2. Performance analysis of various classifiers on audio data

\begin{tabular}{|c|c|c|c|}
\hline \multirow{2}{*}{ Methodology } & \multicolumn{3}{|c|}{ Parameters (\%) } \\
\cline { 2 - 4 } & Accuracy & Precision & Recall \\
\hline SVM & 89 & 89.46 & 90.58 \\
\hline RF & 85 & 88.71 & 87.41 \\
\hline LSTM & 92 & 93.13 & 92.16 \\
\hline BiLSTM & 94 & 94.49 & 93.57 \\
\hline $\begin{array}{c}\text { Proposed } \\
\text { HANN }\end{array}$ & 97 & 96.89 & 97.06 \\
\hline
\end{tabular}

Table 3. Memory usage for various input data

\begin{tabular}{|c|c|c|}
\hline $\begin{array}{c}\text { Types } \\
\text { of Data }\end{array}$ & $\begin{array}{c}\text { Existing } \\
\text { Techniques, } \\
\text { Memory Usage }\end{array}$ & $\begin{array}{c}\text { Proposed HANN } \\
\text { Method's } \\
\text { Memory Usage }\end{array}$ \\
\hline Text & $600 \mathrm{kB}-700 \mathrm{kB}$ & $800 \mathrm{kB}-900 \mathrm{kB}$ \\
\hline Image & $200 \mathrm{MB}-500 \mathrm{MB}$ & $350 \mathrm{MB}-450 \mathrm{MB}$ \\
\hline Video & $800 \mathrm{MB}-1.5 \mathrm{~GB}$ & $750 \mathrm{MB}-1 \mathrm{~GB}$ \\
\hline
\end{tabular}

precision and recall. While comparing with SVM, LSTM and BiLSTM, the existing RF achieved poor performance, i.e. only $79 \%$ of accuracy, $82.15 \%$ of precision and $85 \%$ of recall. The reason is that the percentage for information spilt in the RF are not calculated that leads to low classification accuracy on video data. The neural networks LSTM and BiLSTM achieved better performance than SVM due to its efficiency of hidden layers. However, LSTM and BiLSTM are slower than other activation functions called sigmoid and rectified linear units. To improve the activation layers performance, attention layer mechanism is included in the proposed HANN method that helps to achieve high performance compared to SVM, RF, LSTM and BiLSTM. Therefore, the validation results proved that the proposed HANN achieved $96 \%$ of accuracy, $95.15 \%$ of precision and $96 \%$ of recall on video data.

The next section will discuss the performance of various techniques on audio data.

\subsection{Quantitate analysis of Proposed HANN method on Audio Data}

The audio data about movie reviews are extracted from the collected video data for movie genre classification. Table 2 presents the validated results of different techniques on audio data of Telugu and English movies in terms of accuracy, precision and recall.

The experimental analysis proves that the HANN method has the best performance compared to the other classifiers in terms of accuracy, precision and recall. For instance, HANN method achieved $97 \%$ of accuracy, where RF and LSTM achieved $85 \%$ and $92 \%$ of accuracy on audio data. The SVM technique has only $89 \%$ to $90 \%$ of accuracy, precision and recall on audio data, because the data set has more number of noises, i.e. target classes are overlapping. In addition, SVM is not suitable for large datasets and therefore, SVM shows poor performance than LSTM, HANN and BiLSTM. In addition, the LSTM and BiLSTM are well suited for time-serious data, therefore, they achieved nearly $92 \%$ to $94 \%$ of accuracy, precision and recall. However, the architecture of LSTM and BiLSTM contains only input, output and forget gate, where the proposed HANN uses the encoder and attention layers effectively for movie genre classification that increases high performance than other classifiers.

Table 3 presents the memory usage for existing techniques namely SVM, RF, LSTM and BiLSTM with HANN method on different types of one month collected data. In text' memory usage, the existing techniques uses only $600 \mathrm{kB}$ to $700 \mathrm{kB}$, but HANN method consumed $800 \mathrm{kB}$ to $900 \mathrm{kB}$. The reason is

Table 4. Comparative study of proposed method

\begin{tabular}{|c|c|c|c|c|c|}
\hline Author & Methodology & Input Data Types & $\begin{array}{c}\text { Accuracy } \\
(\mathbf{\%})\end{array}$ & $\begin{array}{c}\text { Precision } \\
(\mathbf{\%})\end{array}$ & Recall (\%) \\
\hline Wi [16] & Gram Layer in CNN & Text & 60.50 & 81.00 & 47.22 \\
\hline $\begin{array}{c}\text { Shambharkar } \\
{[17]}\end{array}$ & DCNN with filtering & $\begin{array}{c}\text { Video Sequence } \\
\text { Data }\end{array}$ & 95.23 & 91.00 & 90.00 \\
\cline { 2 - 5 } & DCNN without filtering & $\begin{array}{c}\text { Video Sequence } \\
\text { Data }\end{array}$ & 90.91 & 85.00 & 82.00 \\
\hline $\begin{array}{c}\text { Proposed } \\
\text { Methodology }\end{array}$ & HANN & Twitter Data & 73.15 & 93.00 & 94.50 \\
\hline $\begin{array}{c}\text { Proposed } \\
\text { Methodology }\end{array}$ & HANN & Video Data & 96.00 & 95.15 & 96.00 \\
\hline
\end{tabular}


that $80 \%$ of information are extracted by using the caption mentioned in twitter data, re-tweets and comments in the collected twitter data for movie genre classification. However, while comparing with other data such as images and video, only limited amount of memory is required for final classification. It proves that the video or image data requires high computation resources and text data consumes less resources that is illustrated in Table 3.

The next section presents discussion on the comparative study between HANN and existing methods.

\subsection{Comparative study}

The performance of the HANN method is compared with the gram layer in CNN [16] and DCNN [17] in terms of accuracy and precision on text and video sequence data.

The results present in the Table 4 indicate that the proposed HANN method has achieve better performance in terms of accuracy and precision when compared with gram layer in CNN [16]. The HANN method has achieved less accuracy (only $73.15 \%$ ) compared to DCNN [17], this is because the HANN method uses the text as input data and the imbalance and skewed data are presented in the collected movie data. However, the HANN method achieved $96 \%$ of accuracy, where DCNN [17] achieved nearly 90-95\% of accuracy on video data. The proposed HANN method effectively pre-process the collected data by using attention layers in neural network. The precision of HANN method has achieved 93\% compared to the both existing techniques, because of the inclusion of the attention layer in the proposed method. The existing Gram layer [16] achieved poor recall (i.e. $47.22 \%$ ), because recall is only calculated for label values and didn't considered the sample values for both input and output data. The existing DCNN has achieved better precision and classification, when considering the filtering operation for final classification. For instance, the DCNN method achieved $91 \%$ of precision with filtering technique and achieved only $85 \%$ of precision without filtering technique. While comparing with DCNN [17], the proposed HANN method achieved $94.50 \%$ of recall values on text data. The DCNN method with filtering technique achieved $90.00 \%$ of recall and achieved only $82.00 \%$ of recall without filtering techniques. The reduction in recall for DCNN method is due to the use of video sequence data that leads misclassification of movie genre.

\section{Conclusion}

Movie Genre Classification is very helpful for the movie recommendation system in the video streaming application. Existing methods involve in analysis of video and audio for the movie genre detection that requires more computational resources. In this study, social media data (i.e. Twitter data related to the Telugu movies) were extracted and provided to the HANN method for the movie genre classification. The proposed HANN method used the relevant information in the encoding process. The other classifiers like SVM, RF, LSTM and BiLSTM were used for performance analysis. The IMDB data were used to evaluate the performance of the HANN method. The proposed HANN method achieved $93 \%$ precision in genre classification, while existing method LSTM achieved $91 \%$ of precision. However, the classification accuracy of the proposed HANN method is less. In future, the accuracy can be improved by implementing feature extraction techniques with the proposed HANN method on both text and other types of data for all movie genre classification as a future work.

\section{Conflicts of Interest}

The authors declare no conflict of interest.

\section{Author Contributions}

The paper conceptualization, methodology, software, validation, formal analysis, investigation, resources, data curation, writing-original draft preparation, writing-review and editing, visualization, have been done by $1^{\text {st }}$ author. The supervision and project administration, have been done by $2^{\text {nd }}$ author.

\section{References}

[1] K. Kundalia, Y. Patel, and M. Shah, "Multi-label Movie Genre Detection from a Movie Poster Using Knowledge Transfer Learning", Augmented Human Research, Vol. 5, No. 1, pp. $11,2020$.

[2] T. G. Hwang, C. S. Park, Hong, J. H., and S. K. $\mathrm{Kim}$, "An algorithm for movie classification and recommendation using genre correlation", Multimedia Tools and Applications, Vol. 75, No. 20, pp. 12843-12858, 2016.

[3] S. M. Daudpota, A. Muhammad, and J. Baber, "Video genre identification using clusteringbased shot detection algorithm", Signal, Image and Video Processing, Vol. 13, No. 7, pp. 14131420, 2019.

[4] P. Muneesawang, L. Guan, and T. Amin, "A New Learning Algorithm for the Fusion of 
Adaptive Audio-Visual Features for the Retrieval and Classification of Movie Clips", Journal of Signal Processing Systems, Vol. 59, No. 2, pp. 177-188, 2010.

[5] S. Vashishtha and S. Susan, "Fuzzy rule based unsupervised sentiment analysis from social media posts", Expert Systems with Applications, Vol. 138, pp. 112834, 2019.

[6] A. C. Pandey, D. S. Rajpoot, and M. Saraswat, "Twitter sentiment analysis using hybrid cuckoo search method", Information Processing \& Management, Vol. 53, No. 4, pp. 764-779, 2017.

[7] K. Pasupa, and T. S. N. Ayutthaya, "Thai sentiment analysis with deep learning techniques: A comparative study based on word embedding, POS-tag and sentic features", Sustainable Cities and Society, Vol. 50, pp. 101615, 2019.

[8] J. Singh, G. Singh, R. Singh, and P. Singh, "Morphological evaluation and sentiment analysis of Punjabi text using deep learning classification", Journal of King Saud UniversityComputer and Information Sciences. Vol. 63, pp. 212-225, 2018.

[9] O. Araque, I. Corcuera-Platas, J. F. SánchezRada, and C. A. Iglesias, "Enhancing deep learning sentiment analysis with ensemble techniques in social applications", Expert Systems with Applications, Vol. 77, pp. 236-246, 2017.

[10] K. Song, H. Kim, Lee, J. and Y. G. Kim, "Dissecting movie performance across multiple distribution channels: An elastic justification theory perspective", Telematics and Informatics, Vol. 35, No. 1, pp. 159-167, 2018.

[11] J. Wei, J. Liao, Z. Yang, S. Wang, and Q. Zhao, "BiLSTM with Multi-Polarity Orthogonal Attention for Implicit Sentiment Analysis", Neurocomputing, Vol. 383, pp. 165-73, 2020.

[12] H. Li, J. Cui, B. Shen, and J. Ma, "An intelligent movie recommendation system through grouplevel sentiment analysis in microblogs", Neurocomputing, Vol. 210, pp. 164-173, 2016.

[13] H.T. Nguyen, and M. Le Nguyen, "An ensemble method with sentiment features and clustering support", Neurocomputing, Vol. 370, pp. 155$165,2019$.

[14] J. Wehrmann and R.C. Barros, "Movie genre classification: A multi-label approach based on convolutions through time", Applied Soft Computing, Vol. 61, pp. 973-982, 2017.

[15] T. Chen, R. Xu, Y. He, and X. Wang, "Improving sentiment analysis via sentence type classification using BiLSTM-CRF and CNN",
Expert Systems with Applications, Vol. 72, pp. 221-230, 2017.

[16] J. A. Wi, S. Jang, and Y. Kim, "Poster-Based Multiple Movie Genre Classification Using Inter-Channel Features", IEEE Access, Vol. 8, pp. 66615-66624, 2020.

[17] P. G. Shambharkar and M. N. Doja, "Movie trailer classification using deer hunting optimization based deep convolutional neural network in video sequences", Multimedia Tools and Applications, 2020.

[18] S. Gao, M. T. Young, J. X. Qiu, H. J. Yoon, J. B. Christian, P. A. Fearn, G. D. Tourassi, and A. Ramanthan, "Hierarchical attention networks for information extraction from cancer pathology reports", Journal of the American Medical Informatics Association, Vol. 25, No. 3, pp. 321-330, 2018. 